PROCEEDINGS OF THE

AMERICAN MATHEMATICAL SOCIETY

Volume 126, Number 10, October 1998, Pages 3071-3074

S 0002-9939(98)04472-4

\title{
LAYERED CIRCLEPACKINGS AND THE TYPE PROBLEM
}

\author{
RYAN SIDERS
}

(Communicated by James West)

\begin{abstract}
We study the geometric type of a surface packed with circles. For circles packed in concentric layers of uniform degree, the circlepacking is specified by this sequence of degrees. We write an infinite sum whose convergence discerns the geometric type: if $h_{i}$ layers of degree 6 follow the $i$ th layer of degree 7 , and the $i$ th layer of degree 7 has $c_{i}$ circles, then $\sum \log \left(h_{i}\right) / c_{i}$ converges/diverges as the circlepacking is hyperbolic/Euclidean. We illustrate a hyperbolic circlepacking with surprisingly few layers of degree $>6$.
\end{abstract}

\section{INTRODUCTION}

A circlepacking is a collection of non-overlapping circles, each of which meets its 7 or fewer neighbors tangentially, so that every point is in an area bounded by 3 or fewer circles. All the results of this paper are true if 3 and 7 are replaced with larger numbers, but we can't prove anything without some universal bounds. In particular, we examine a layered circlepacking: a central circle is surrounded by 6 (say) neighbors, which form the first layer. Around these six wraps a layer of 12 (say) circles so that each circle of the first layer has degree 7. Around each layer the next layer wraps so that all circles in a layer have the same degree. The next layer has 1 or 2 times as many circles as the previous layer, as the degree of the circles in the previous layer is 6 or 7 . The degrees of the successive layers are a sequence of 6's and 7's. For which sequences does the circlepacking pack hyperbolic space, and for which does it pack Euclidean space? If we draw the circles in the Euclidean plane, the question is: for which sequences will the limiting figure be finitely or infinitely large?

A circlepacking packs a hyperbolic/Euclidean geometry as a random walk on the circles is transient/recurrent; this is proven in [3] and [5]. This important computation shows the circles are packed evenly enough to capture the surface's geometry in their combinatorics. It assumes that the circles pack the surface evenly enough to escape a topological condition: we assume that finitely many circles meet each bounded subset of the surface. We shift our viewpoint from random walks to electronics via a more formal equivalence. A random walk on a graph (starting at point $p$ ) is transient/recurrent as an electric flow (into point $p$ ) meets finite/infinite

Received by the editors November 28, 1995 and, in revised form, February 28, 1997.

1991 Mathematics Subject Classification. Primary 52C15.

Key words and phrases. Circlepacking, electric network.

This work was done under Dr. Phil Bowers of Florida State University during FSU's 1994 Research Experience for Undergraduates. Dr. Bowers was an inspiring mentor. I will treasure what I learned from our conversations. The program was sponsored by the NSF.

(C)1998 American Mathematical Society 
resistance because the finite difference equations for these two processes are the same. A direct analogy between the geometric type of a surface and electric flow on a grid which is assumed to partition the surface evenly is drawn in [1] and [2]. Some nice, general computations for the resistance of an infinite electric grid appear in $[4]$.

The resistance in a uniform Euclidean grid (such as the the triangular grid corresponding to the penny packing of Euclidean space) is asymptotically logarithmic. The finite difference equation for voltage (the voltage at a point is the average of the voltages at its neighbors) limits, if we examine successively larger sections of a regular grid, to the continuous Laplacian equation for electric flow on a continuous surface (that voltage at a point is the average of voltages in a circle around it), whose solution is logarithmic. (In $n$ dimensions, the potential is $1 / r^{n-1}$, so the voltage is $\int 1 / r^{n-1}=\log$ for $n=2$. In higher dimensional Euclidean space, the resistance to infinity is finite, so we cannot tell $3 \mathrm{D}$ sphere packings' geometry with random walks or current. What is to be done?) In particular, the resistance from the apex of a triangular mesh to a point on the opposite side is a logarithmic function $\alpha \log (n)$ of the side length $n$. $\alpha$, as a function of position along the faraway side of a triangular mesh, limits to some function on $[0,1]$. Does it tend towards $\infty$ at 0 ? No, it is universally bounded by some $\alpha_{0}$.

Consider a circlepacking in which $h_{i}$ layers of degree 6 follow the $i$ th layer of degree 7 . The graph of this circlepacking (a node represents a circle; an edge indicates that circles are neighbors) contains the graph in Figure 1, which is electrically equivalent to the tree in Figure 2. The wires have resistance $\alpha \log \left(h_{i}\right) \leq \alpha_{0} \log \left(h_{i}\right)$; each node gives rise to $h_{i}$ wires. The resistance in a tree with $h_{i}$ branches of resistance $r_{i}$ is $\sum_{i} r_{i} / \Pi_{k<i} h_{k}$, so our tree has resistance (less than) $\alpha_{0} \sum \log \left(h_{i}\right) / \Pi_{k<i} h_{k}$. If this tree has finite resistance, then a positive current reaches infinity along it, and the original circlepacking must have been hyperbolic.

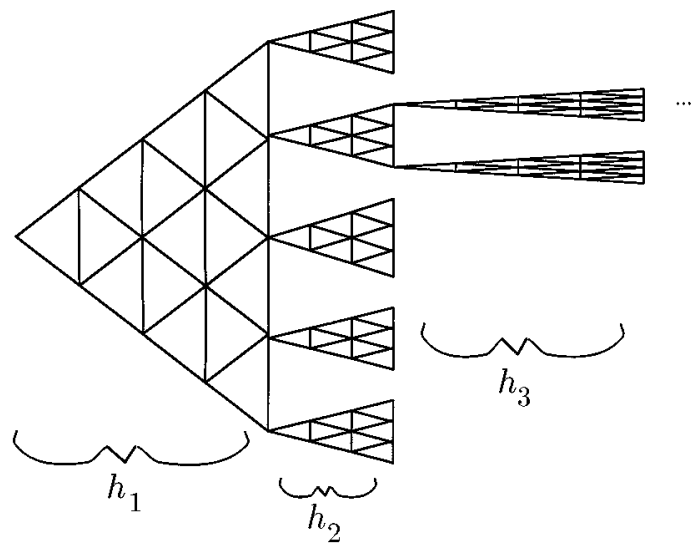

Figure 1

For which sequences $\left\{h_{i}\right\}$ is $\sum \log \left(h_{i}\right) / \Pi_{k<i} h_{k}$ finite? If $h_{i}$ is constant, the sum is a convergent geometric series. If $h_{i}=2^{i}$, resistance grows as $\sum i / 2^{i^{2}}$, which converges faster. (If one sequence $h$ grows faster than another $h^{\prime}$, then the sum for $h^{\prime}$ will converge faster than the sum for $h$, until $h$ suddenly grows so fast that the sequence stops converging. This seems more intuitive if we phrase it: as a 


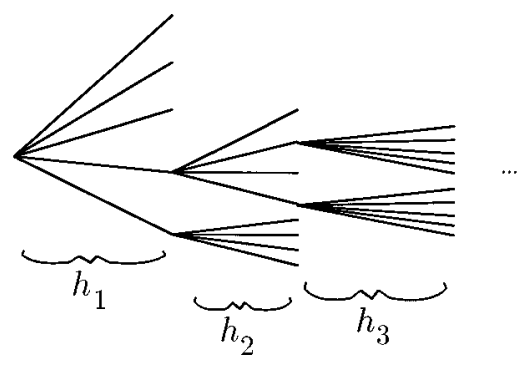

FiguRe 2

circlepacking gets closer to being hyperbolic, its resistance gets concentrated in its first few terms; i.e. near its center. Or: as a hyperbolic circlepacking gets close to being Euclidean, more and more of its resistance is found far from the center.) If $h_{i}$ is the $i$-fold composition of the exponential function, the series will converge. But if $h_{i}$ is the $2 i$-fold composition of exp, then the series will diverge.

Finally, we want to see that if $h_{i}$ grows too rapidly, then the circlepacking really is Euclidean, rather than just failing our test for hyperbolicity. Let's allow current to flow without resistance within any layer; this will decrease the resistance and simplify the graph so that we can compute its resistance. The number of wires between layers grows linearly between layers of degree 7 , and the growth rate is no more than twice the number of circles in the previous layer of degree 7. See Figure 3 . The resistance between the $i$ th and the $i+1$ th layers is

$$
\sum_{k=1}^{h_{i}} 1 /(1+2 k) c_{i}>\log \left(h_{i}\right) / c_{i} .
$$

The number of circles in the $i$ th layer is $c_{i}>2^{i} \Pi_{k<i} h_{i}$. So the net resistance is $>\sum \log \left(h_{i}\right) / 2^{i} \Pi_{k<i} h_{i}$. For $h_{i}$ the $2 i$-fold composition of the exponential function, the resistance is infinite, and so the surface is Euclidean.

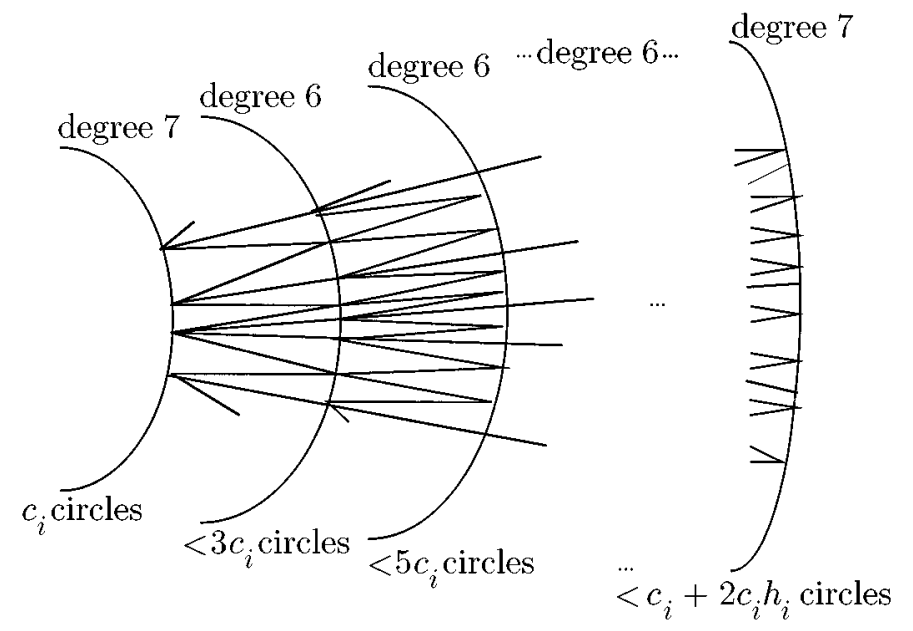

Figure 3 


\section{REFERENCES}

[1] Doyle, P, On deciding whether a surface is hyperbolic or parabolic, Contemporary Mathematics, Vol 72, 1988. MR 89h:53043

[2] Doyle, P, and Snell, L Random Walks and Electric Networks, MAA (Carus Monograph Series), 1984. MR 89a:94023

[3] He, Z X, and Schramm, O, Hyperbolic and Parabolic Packings, preprint.

[4] Lyons, R, Random Walks and Percolation on Trees, Annals of Probability, Vol 18, p. 931-958, 1990. MR 91i:60179

[5] McCaughan, G, Transience and Recurrence, Proceedings of the AMS, to appear.

Department of Mathematics, Princeton University, Princeton, New Jersey 08544

E-mail address: rcsiders@math.princeton.edu 